\title{
A three-dimensional muscle-based facial expression synthesizer for model-based image coding
}

\author{
Yuong-Wei Lei*, Ja-Ling Wu, Ming Ouhyoung \\ Communications and Multimedia Laboratory, Department of Computer Science and Information Engineering, \\ National Taiwan University, Taipei, Taiwan, $R O C$
}

Received 22 August 1994

\begin{abstract}
A three-dimensional muscle-based facial expression synthesizer is proposed. The proposed synthesizer will compute the contraction of 19 muscles and rotation of the jaw from 22 feature points estimated by the analyzer, then apply the muscle contraction model to modify the $3 \mathrm{D}$ head model. The head motion, including translation and orientation, is also derived from a set of threc fcaturc points. Currently the analyzer is implemented on a PC with a camcorder with near real-time performance, and the synthesizer receives the feature points from the analyzer and synthesizes facial expressions on an SGI Indigo in real time.
\end{abstract}

Keywords: Model-based image coding; Facial expression synthesizer; Virtual human

\section{Introduction}

The model-based analysis synthesis image coding (MBASIC) scheme, first proposed in $[1-3,8]$, employs human face visual information processing for communication tasks. On the one hand, the computer vision and pattern recognition side concerns monitoring the movement of features and variation of attributes as if the computer has eyes. On the other hand, the computer graphics side concerns the reconstruction of expressive and realistic facial expression. However, the link between the analyzer and the synthesizer is still vague in many aspects. Few concentrate on investigating

\footnotetext{
* Corresponding author.
}

what the synthesizer requires and what the analyzer can offer.

In $[10]$ the authors have proposed an automatic feature point extractor based on image processing operations and have pointed out the direction of an analyzer-synthesizer system. This paper will describe the current version of the system with an emphasis on the synthesizer part. We have developed a $3 \mathrm{D}$ head motion estimator and a $3 \mathrm{D}$ feature points estimator, with the $2 \mathrm{D}$ feature points generated by the extractor as input. For the human face synthesizer, we present a muscle-based facial expression generator that computes the contraction of muscles and rotation of jaw from the estimated 3D feature points. A 3D head model is then modified according to Waters' muscle model [20] and the rotation of the jaw. 


\section{Issues in model-based analysis synthesis image coding}

Before listing the problems one will encounter when developing an analyzer-synthesizer pair, we summarize the data processing flow of an MBASIC system. In the encoder phase, the encoder analyses the input image to gather geometric parameters of the human body. The gathered geometric parameters, including feature points on eyebrows, eyes, eyelids, nose, and mouth, can be used to generate the so-called facial expression. The more detailed and abstract information an analyzer can offer, the more impressive image the decoder, which is a model synthesizer, can generate.

Computer vision and computer graphics are dual domains of the MBASIC system, the relation can be modeled as dual pyramids [18]. The synthesizer, in the decoder phase, will generate a virtual human [12] according to the received parameters. The facial expression is reconstructed to reproduce the emotion and feeling of the speaker. The virtual human is usually generated from a $3 \mathrm{D}$ model that is a common data set shared by both the analyzer and synthesizer using parametric patches or a polygon set with about $400-3000$ patches. The synthesizer adjusts the $3 \mathrm{D}$ head model by various facial expression methods and, for photorealistic images, applies facial texture mapping on the model, resulting in a synthesized image resembling the original speaker's face both in structure and expression.

\subsection{The feature point extractor}

The parameters a feature point extractor analyzes include head position, orientation, and feature points position on eyebrows, eyes, eyelids, eyes, nose and mouth outline with respect to a frame attached on the head. In one strategy, the parameters points are marked up in the first leading image manually, and then roughly tracked by block matching in the succeeding frames. Other strategies employ magnetic sensors and optical fibers for measuring position and kinetic motion, or by tracking small round adhesive bright spots on the face. Although computation efficiency may be achieved, the above manual methods are sometimes uncomfortable and inadequate.

A desired methodology is to apply digital image processing on the input image, then $3 \mathrm{D}$ motion parameters are estimated after the $2 \mathrm{D}$ motion is either analyzed or estimated. The analysis should be autonomous and robust, with as few assumptions as possible. In a step by step manner, the extraction can proceed in two steps: first estimate the head motion and then extract the feature points on the face.

Many extraction methods based on image processing have been proposed to estimate $2 \mathrm{D}$ information successfully. On estimating 3D parameters from 2D information. Ohmura [17] used three marks adhered to a face in a triangular configuration, and then estimated the $3 \mathrm{D}$ position of the marks using distances between the marks. Skita [6] and Suenaga [15] used centroid points of the head and face area to calculate the orientation of the head. A neural network method was proposed by Fukuhara [9], who used a three-layer model and a training set comprising 397 possible motion patterns.

In the feature points extraction step, sometimes called the Face Reader [14], most of the proposed methods utilize the characteristic chrominance and luminance properties of the human face and an empirical knowledge base. In the direct extraction method, the possible position of the organs can be estimated with the face region as reference and with the knowledge provided by the empirical data set [10]. Although the direct extraction method will suffer from environmental conditions, such as bad lighting and the background scene, and will also suffer in the case of unexpected head orientation, there are two reasons that support this approach. First, consider the applications such as videophone, the lighting and background scene seldom change and the speaker will usually look in the camera direction. Second, it is efficient and can even be implemented on a PC-based platform. Many methods have been proposed based on the direct extraction method, such as $[4,9]$. 


\subsection{The facial expression synthesizer}

A variety of excellent research on human face image generation has been done in recent years. The facial action coding system (FACS) [7] describes the set of all possible basic actions performed on a human face. Waters [21] proposed a muscle model that is independent of the underlying bone structure and uses the control parameters based on FACS. Terzopoulos [19] has extended Waters' model, using three-layered deformable lattice structures for facial tissue. The three layers correspond to skin, subcutaneous fatty tissue, and muscles. The physics-based graphics modeling methods are employed in a synthesizer because moving only the specific feature points on the 3D shape model to the desircd position will not meet the realism requirements. This is because the translation of a vertex is not linear with respect to the movement of feature points. The facial expression generator is somewhat overlaid with the facial animation field in computer graphics, but different in that a synthesizer considers under which parameter configuration the feature points will get to the desired position and whether the interpolations of nonfeature points are realistic.

\subsubsection{The 3D shape model}

The first issue of generating a speaker-like virtual human is to create a $3 \mathrm{D}$ model of a specific person. This model must be a head model that can supply geometrical information for each organ. One possible method is to create a face model from multiple view images, of course the number of views are as few as possible. Kurihara [11] and Thalmann [13] used multiple photographs to reconstruct the 3D model of a face using interpolation in 2D cylindrical space. Akimoto [5] used a dynamic programming method to extract the feature points on the profile of a face. The position data allow the eyes, nose, and mouth to be extracted from front images effectively. Then the 3D model of the person is created by modifying the generic head model by using the extracted features.

\subsubsection{Facial texture mapping}

The facial images generated from the traditional rendering method (e.g., interpolated polygon shading) tend to look rather artificial and cartoonlike. The shading model for human skin is still under investigation and has produced few results. So the texture mapping technique, especially using the original speaker's images as texture, is the most popular way. However, because there are geometrical constraints between each 3D model patch and a specific region on the texture map, merely projecting the image on the face model is not good enough. Nahas [16] employed a registration between each patch and a particular region manually. Waters [21] used an adaptive meshing technique on a radial range map that modifies the mesh according to the magnitude of the gradient of the range map. Kurihara [11] used the information obtained from 3D model construction from multiplc photographs to compose the photographs into one image using a weight function.

\section{An example MBASIC system}

A complete MBASIC system implementation is proposed in this section. The analyzer with automatic feature points extraction capability has been proposed in $[10]$. This paper will focus on the synthesizer implementation issues.

\subsection{Review of the PFPE}

The processing pipcline of the preliminary feature point extractor (PEPE) is shown in Fig. 1 and is summarized as follows. More detailed description can be found in [10].

We make some reasonable assumptions as necessary conditions of the input image:

(a) The user's face must appear at about the center of the input inage.

(b) The area of the user's face is at least onesixteenth the size of the input image.

(c) The eyebrows, eyes, and mouth must be visible.

(d) The user cannot wear sunglasses or glasses with a black frame.

(e) The angle of the major axis and the perpendicular line of the user's face cannot be greater than $90^{\circ}$. 


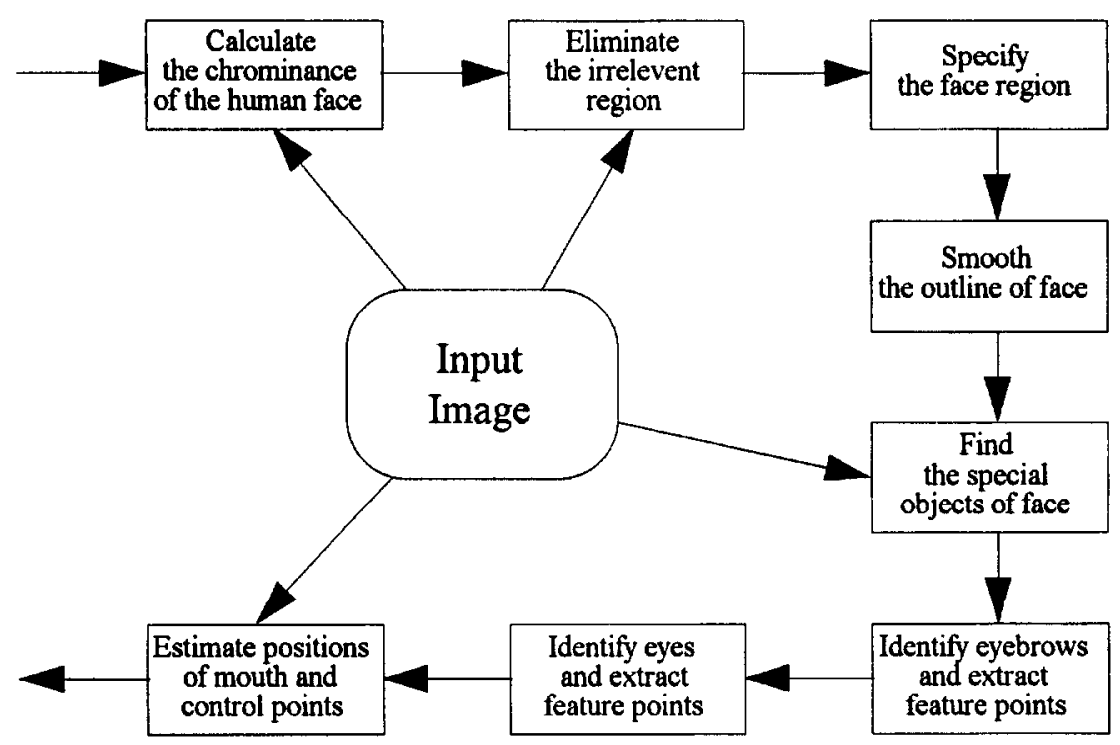

Fig. 1. The flowchart of the PFPE.

1. The average chrominance and luminance of the center one-sixteenth area of the input image are calculated as those of a human face.

2. Thresholding is applied on the input image to remove the irrelevant region, using the average chrominance value in the previous step. The object that contains the largest amount of adjacent residual pixels is selected as the human face region. Then image processing operations are employed to fill up the holes inside the face region, fill up the hollows of the contour, and smooth the outline of the face.

3. The special areas of the face region are specified as candidates of special objects according to the empirical luminance value.

4. The highest special object is chosen as the higher eyebrow. The lower eyebrow is picked depending on the axial information from the higher eyebrow.

5. The eyes are extracted in a similar strategy as the eyebrows.

6. The nose, nostrils, and mouth outline are estimated by empirical knowledge.

The feature points extracted by the PEPE are shown in Fig. 2. We will use the denotation of Fig. 2 in the following discussion.

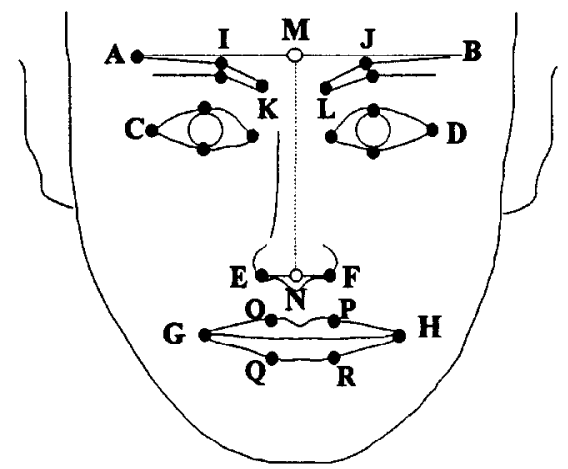

Fig. 2. The control points of a human face. The filled dots are the feature points extracted by PFPE. $M$ is the middle point of $\bar{A} \bar{B}$ and $N$ is the middle point of $\overline{E F}$.

\subsection{The preliminary facial expression synthesizer}

In the decoder phase, the preliminary facial expression synthesizer (PFES) will first estimate 3D head motion and 3D feature points from the 2D feature points generated by the PFPE, and then generate the human face by the muscle-based facial expression generator. 


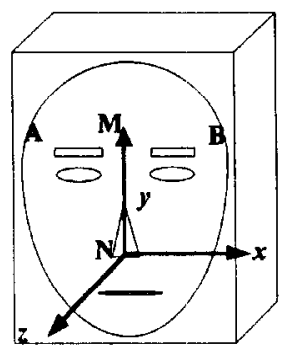

(a)

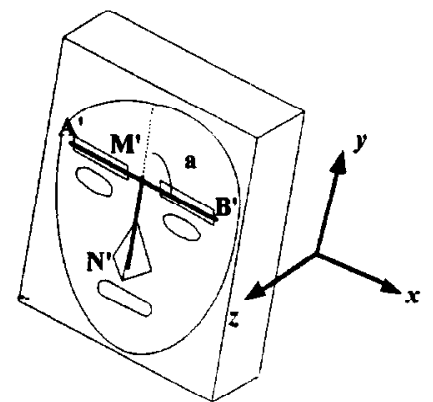

(b)

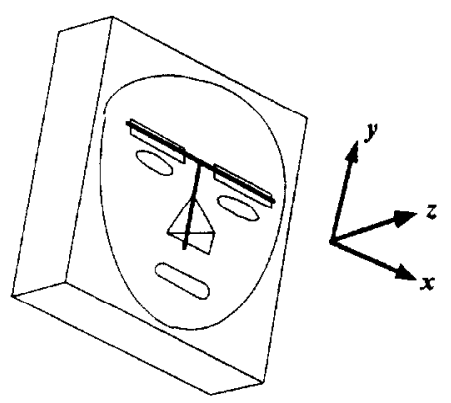

(c)

Fig. 3. Estimated head motion from $\overline{A B}$ and $\overline{M N}$ in parallel projection. (a) A 3D coordinate system attached on face. (b) From the length of $\overline{A B}$ and $\overline{M N}$ and angle $a$, the transfromed coordinate frame can be estimated. (c) The other possible coordinate frame under the same configuration as in (b).

\subsubsection{Head reader}

The idea of head motion estimation is similar to Ohmura et al. [17] but using feature points instead of manually attached identification marks. To solve the motion estimation problem, we require the precise measures of the following lines: $\overline{A B}, \overline{C D}$, $\overline{E F}$, and $\overline{M N}$, which can be obtained from positions of the feature points $A, B, C, D, E$, and $F$ in the front view image. Then the problem to be solved is how to make the position calculation robust enough from the nonrigid feature points.

Consider a three-dimensional coordinate system attached on the face with the $x$ axis pointing to the right, the $y$ axis upward and the $z$ axis outward (Fig. 3(a)), then $\overline{A B}$ is parallel to the $x$ axis and $\bar{M} \bar{N}$ to the $y$ axis. If we can calculate the transformed coordinate system from the transformed $\overline{A B}$ and $\overline{M N}$, in turn we can get the translation and orientation of the head (Fig. 3(b)).

Figs. 3(b) and (c) depict the two possible conditions for the same feature points configuration $\left(A^{\prime}, B^{\prime}, M^{\prime}, N^{\prime}\right)$, Fig. 3(b) is slightly looking down and left, but Fig. 3(c) is looking up and right. Under parallel projection, it is indeed very difficult to distinguish unless other information such as ears and eyes is provided. However, in perspective projection, $\overline{A M} \neq \overline{M B}$ if the head is not exactly facing front, and $\overline{A M}>\overline{M B}$ if $A$ is closer to the camera's position. Let us give a detailed derivation below. We choose three feature points to calculate the head motion, and these three points are the left and right eyebrow outer endpoints $(A, B)$ and the central point of the nostrils $(N)$.

Let $B=\left(x_{1}, y_{1}, z_{1}\right), N=\left(x_{2}, y_{2}, z_{2}\right)$, and $M=$ $\left(x_{3}, y_{3}, z_{3}\right)$, where $M=(A+B) / 2$, then after perspective projection, the screen coordinates of $B$, $N$ and $M$ become

$$
\begin{aligned}
& B^{\prime}=\left({ }^{1} P_{x},{ }^{1} P_{y}\right)=\left(\frac{D x_{1}}{z_{1}}, \frac{D y_{1}}{z_{1}}\right), \\
& N^{\prime}=\left({ }^{2} P_{x},{ }^{2} P_{y}\right)=\left(\frac{D x_{2}}{z_{2}}, \frac{D y_{2}}{z_{2}}\right), \\
& M^{\prime}=\left({ }^{3} P_{x},{ }^{3} P_{y}\right)=\left(\frac{D x_{3}}{z_{3}}, \frac{D y_{3}}{z_{3}}\right),
\end{aligned}
$$

where $D$ is the distance from eye to screen in an ideal projection model. Now we have

$$
\begin{aligned}
& D x_{1}={ }^{1} P_{x} z_{1}, \\
& D y_{1}={ }^{1} P_{y} z_{1}, \\
& D x_{2}={ }^{2} P_{x} z_{2}, \\
& D y_{2}={ }^{2} P_{y} z_{2}, \\
& D x_{3}={ }^{3} P_{x} z_{3}, \\
& D y_{3}={ }^{3} P_{y} z_{3},
\end{aligned}
$$


and the lengths of $\overline{M N}, \overline{B N}$ and $\overline{B M}$ are not changed in $3 \mathrm{D}$ :

$$
\begin{aligned}
& \left(x_{3}-x_{1}\right)^{2}+\left(y_{3}-y_{1}\right)^{2}+\left(z_{3}-z_{1}\right)^{2}=\overline{B M}^{2}, \\
& \left(x_{3}-z_{2}\right)^{2}+\left(y_{3}-y_{2}\right)^{2}+\left(z_{3}-z_{2}\right)^{2}=\overline{M N}^{2}, \\
& \left(x_{1}-x_{2}\right)^{2}+\left(y_{1}-y_{2}\right)^{2}+\left(z_{1}-z_{2}\right)^{2}=\overline{B N}^{2} .
\end{aligned}
$$

Now there are nine unknowns and nine equations, we can solve the position of $B, M$ and $N$. But since the equations are not all linear, sometimes we will get multiple solutions, in a situation similar to Fig. 3. In such a case, the length of $\overline{A M}$ is used as a reference versus the length of $\overline{B M}$ to choose the correct solution from possible ones. In general, we are free to choose other feature points. For example, the eye endpoints instead of the eyebrow endpoints can be used. Therefore, multiple sets of data can be used in PFES to estimate the possible values and the averaged one is assumed to be the answer. In this way, the head motion estimation should be more accurate.

\subsubsection{Facial expression generation}

The three-dimensional muscle-based method from Waters [20] is used for generating a realistic facial expression. We use a 20-parameter system (19 for muscles and one for jaw rotation) that calculates the calibrated contraction and jaw rotation parameters from the $3 \mathrm{D}$ feature points.

In the first step, the 2D feature points must be transformed into 3D feature points in the head model. The positions of the $3 \mathrm{D}$ feature points are estimated from the 2D feature points by linear decomposition, as shown in Fig. 4. Each point is decomposed as a linear combination of two unit vectors along transformed $\overline{A^{\prime} B^{\prime}}$ and $\overline{M^{\prime} N^{\prime}}$ (Fig. 4, left) in $2 \mathrm{D}$ space. Then the $3 \mathrm{D}$ feature points in the front view image can be located by the coefficients $v, u$ with respect to $\overline{A B}$ and $\overline{M N}$ (Fig. 4 , right). Of course, the linear decomposition does not hold under perspective projection. However, the results are close enough.

The next step is to calculate the contraction of muscles from the translation vectors of feature

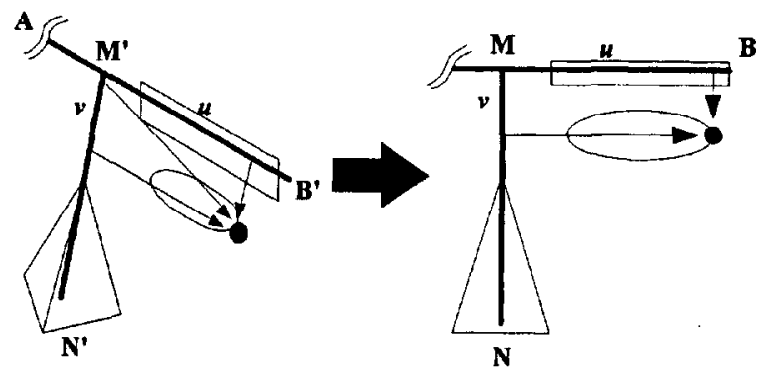

Fig. 4. Estimated 3D feature points from 2D feature points. The linear combination coefficients $v, u$ are used in the front view image.

points. We have extended Waters' muscle model, as shown in Fig. 5(a):

$x^{\prime} \propto f(K, A, R, x)$,

$y^{\prime} \propto f(K, A, R, y)$,

where

$A=(\cos (m / p * p / 2))^{\mathrm{Ca}}$,

$R=$

$\begin{cases}(\cos ((1-D / \mathrm{Rs}) * p / 2))^{\mathrm{Cr}} & \text { if } D<\mathrm{Rs}, \\ (\cos ((D-\mathrm{Rs}) /(\mathrm{Rf}-\mathrm{Rs}) * p / 2))^{\mathrm{Cr}} & \text { if } \mathrm{Rs}<D<\mathrm{Rf} .\end{cases}$

The factor $K$ and the newly introduced factors $\mathrm{Ca}, \mathrm{Cr}$ are muscle-specific and range from 0.25 to 4.0. With the above formulas, consider a feature point $P$ that is affected by only one muscle (Fig. 5(a)), the weight $w$ can be determined through (3.10) and (3.11). Then the quantity of the contraction can be calculated by

$\frac{\mid \text { translation vector } \mid}{\text { weight } w}$

If $P$ is affected by two muscles (Fig. 5(b)), the translation vector can be decomposed into two vectors parallel to the direction from $P$ to the bone attachment point of each muscle. Then the contraction on each direction can be calculated individually. From the 3D feature points position, calibrated estimates are computed in the following. 


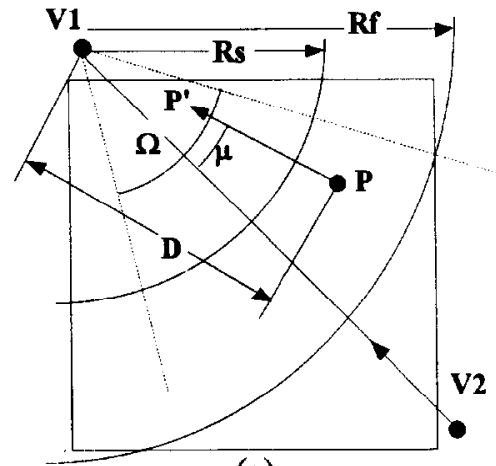

(a)

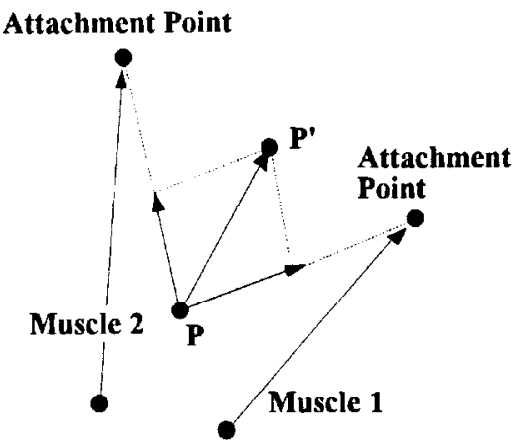

(b)

Fig. 5. Computing contraction from translation vector of feature point. (a) The FP is affected by only one muscle. (b) When affected by two muscles, a linear combination is used.

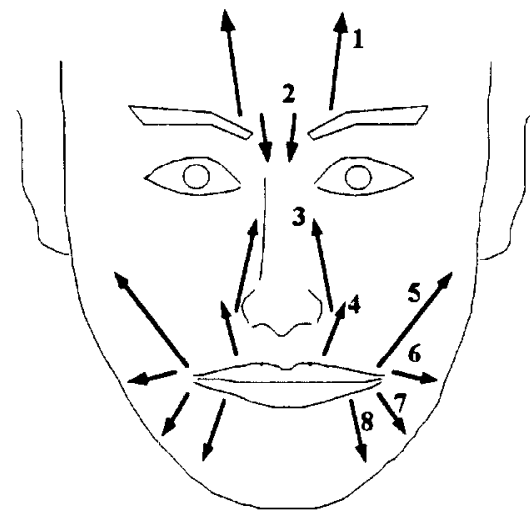

1. Frontalis

2. Corrugator

3. Levator labii superioris alaeque nasi

4. Levator labii superioris

5. Zygomaticus major

6. Buccinator

7. Depressor labii inferiors

8. Depressor anguli oris

Orbicularis oculi and Orbicularia oris are not shown here

Fig. 6. Muscle configuration in the PFES.

Fig. 6 shows the geometrical relation of feature points (FP) and muscles.

1. From the endpoint of lips $(G, H)$ (see Fig. 2) to estimate the contraction of muscle \#5 (zygomaticus major), muscle \#6 (buccinator) and muscle \#7 (depressor labii inferiors).

2. Contraction of muscle \#4 (levator labii superiors) from the under lip FP $(O, P)$.

3. Contraction of muscle \#8 (depressor anguli oris) from the lower lip $\operatorname{FP}(Q, R)$.

4. Contraction of muscle \#3 (levator labii superioris alaeque nasi) from nostrils $(E, F)$.

5. Contraction of muscle \#2 (corrugator) from the inner FP on eyebrows $(K, L)$.
6. Contraction of muscle \#1 (frontalis) from the middle and end $\mathrm{FP}$ on eyebrows $(A, B, I, J)$.

7. The vertical length of the chin relating $\overline{M N}$ determines the rotation of the jaw. Because the chin outline is hardly extracted clearly in the current version of PFPE, the result is not stable.

\subsection{Experimental results and discussion}

Several photos of the results are shown below. Fig. 7(a) shows the input image captured by a camcorder. Fig. 7(b) shows the image when pasting the 


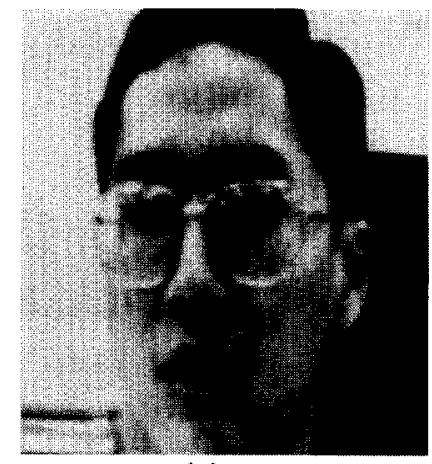

(a)

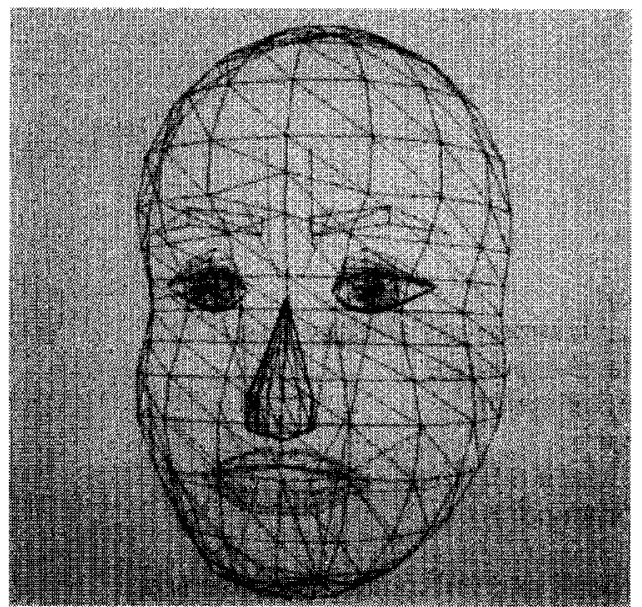

(c)

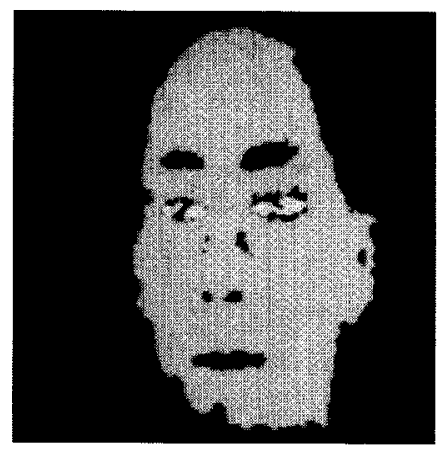

(b)

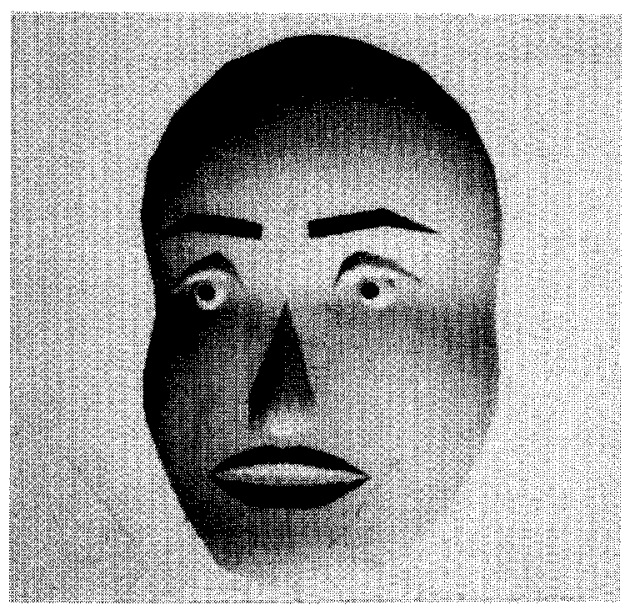

(d)

Fig. 7. Experimental result images. A man is wearing a pair of glasses with silver border.

eyebrows and eyes using the feature points of the face that are extracted by the PFPE. Fig. 7(c) shows the wire-frame model that simulates the speaker's face, the thin blue lines represent muscles of the face. Fig. 7(d) shows the image in Gouraud shading without texture mapping. In this experiment, the speaker wears a pair of glasses with no obvious expression, the simulated expression does look like the speaker. In the second experiment the speaker makes a grimace in Fig. 8(a). In Fig 8(d), although the feature points are located at the right position, the result image incorrectly shows an emotion of sad. The mismatch shows that the characteristic of the grimace cannot fully be represented by 22 feature points alone. The humping around the mouth, for example, is a significant factor in the grimace, but currently no information in our muscle model is coded for this feature. The choice of feature points has been widely studied in the literature [2], but the employment of other feature attributes, such as skin deformation, is still an open problem. As the last experiment, Fig. 9 shows a test frame for one of the authors who is bearded and wearing glasses, of course, our 3D face model does not contain glasses.

The PFES was implemented on a SUN Sparcstation 10 and an SGI Indio ${ }^{2}$ based on the $X$ windows environment. On Sparcstation 10 our system supports real-time wire frame and near real-time flat shading, while on the Indigo ${ }^{2}$ our system supports 


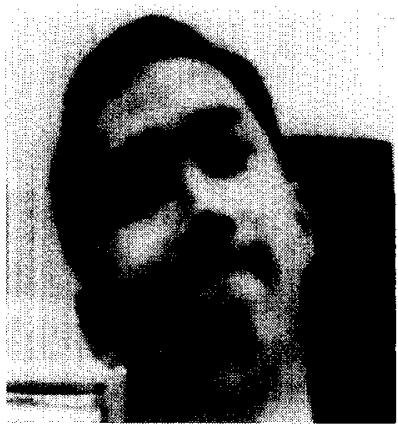

(a)

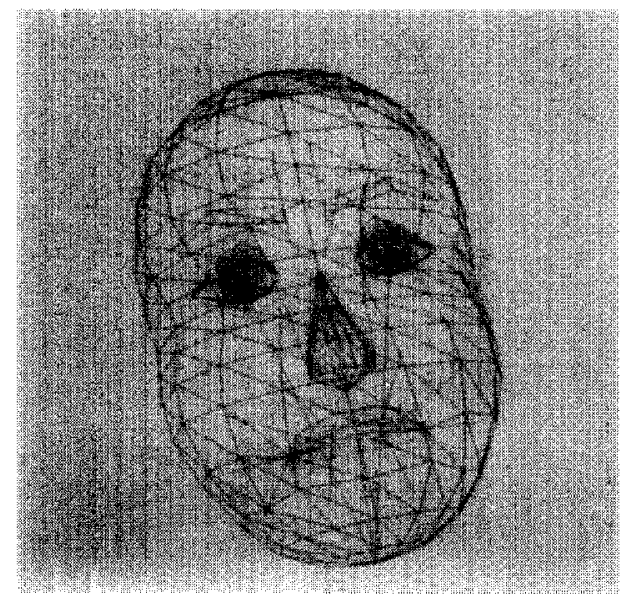

(c)

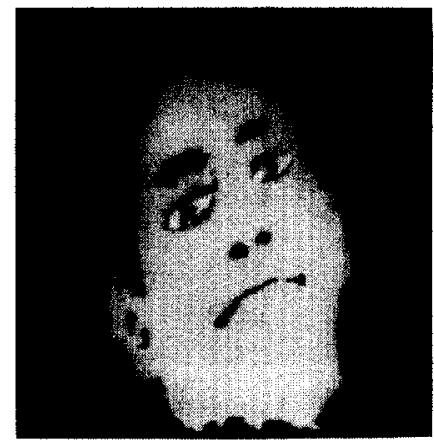

(b)

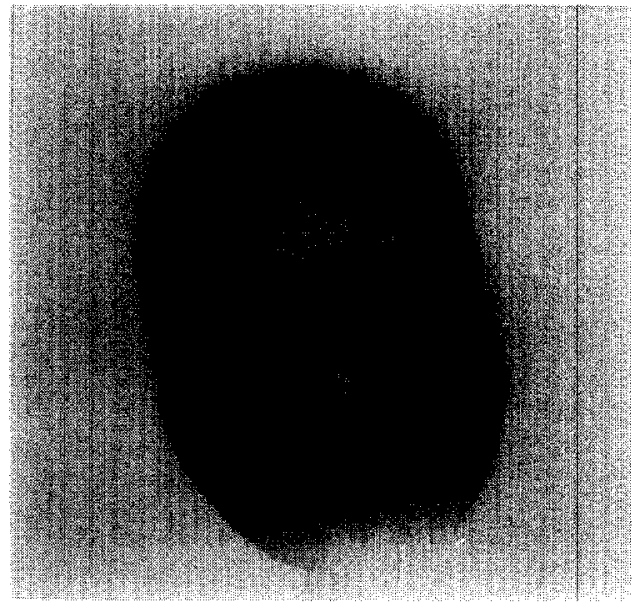

(d)

Fig. 8. An example of estimation error in which the user makes grimaces but the simulated expression shows an emotion of sad.

real-time wire frame and Gouraud shading. Instead of allocating a real data/phone line to transmit the coded parameters, in the current version the estimates from PFPE are transferred directly to the PFES via LAN.

\section{Conclusions}

A preliminary version of the FES is proposed and developed. The proposed PFES can automatically generate expressive face images based on 22 estimated feature points from the earlier proposed PFPE [10]. The head motion of transla- tion and orientation is also derived from a set of three feature points. The experimental results show that the PFES can simulate the speaker's expression effectively in the case that the speaker does not make bizarre faces. In fact, the PFES can generate emotion expressions by itself, for example, smile, fear, sad, anger, disgust and laugh, but when generated from the feature points, it seems to include less emotion of the speaker. Texture mapping from real human photos is still under development, and a more robust and reasonable method for computing contraction of the muscles from the feature points would be future work. 


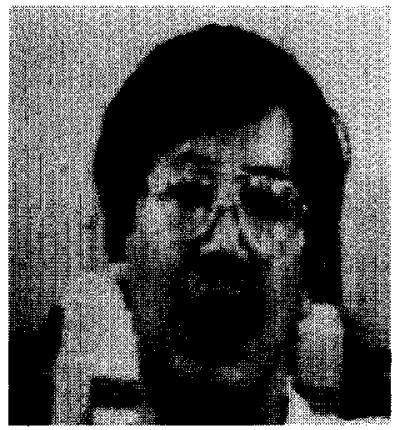

(a)

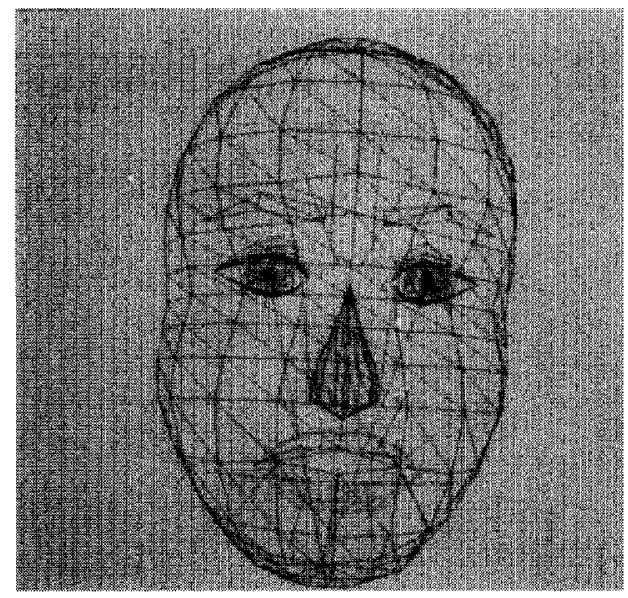

(c)

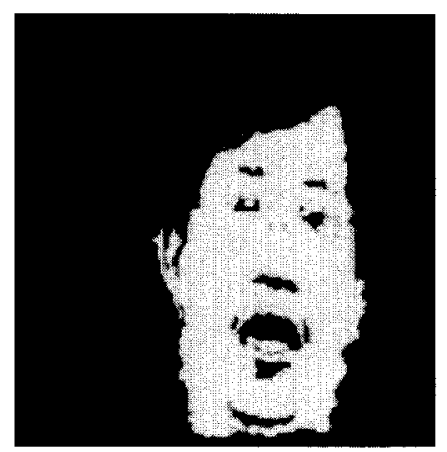

(b)

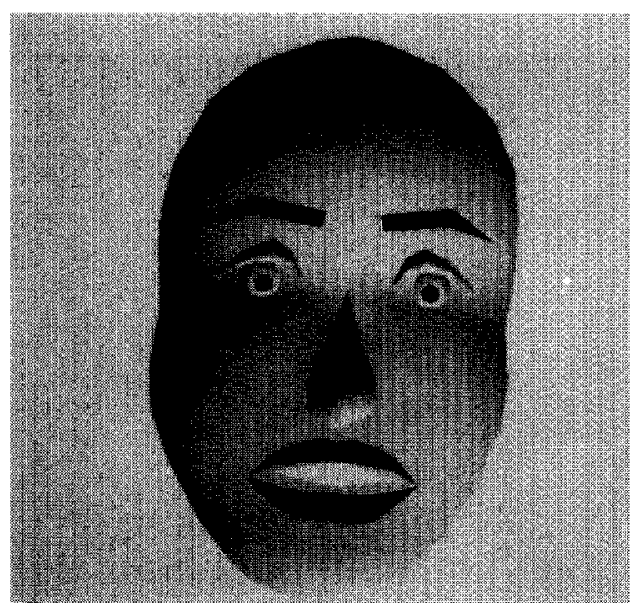

(d)

Fig. 9. One of the authors is bearded and wearing glasses.

\section{Acknowledgements}

The authors would like to thank the anonymous reviewers for their useful comments.

\section{References}

[1] K. Aizawa, H. Harashima and T. Saito, "Model-based analysis synthesis image coding of videophone images: Conception and basic study of intelligent image coding", Trans. ICICE, Vol. E72-5, 1989.

[2] K. Aizawa, H. Harashima and T. Saito, "Model-based analysis synthesis image coding (MBASIC) scheme for a person's face", Signal Processing: Image Communication, Vol. 1, No. 2, October 1989, pp. 139-152.
[3] K. Aizawa, T. Saito and H. Harashima, "Construction of a 3-dimensional personal face model for knowledge-based image data compression", Nat. Conf. Record IEICEJ, Musashino, Japan, 3-6 September 1986, pp 1-221.

[4] S. Akamatsu, T. Sasaki and H. Fukamachi, "An accurate and robust face identification scheme", Proc. 1/th IAPR Internat. Conf. on Pattern Recognition, 1992.

[5] T. Akimoto and Y. Suenaga, "3D facial model creation using generic model and front and side views of face", IEICE Trans. Inform. Systems, Vol. E75-D, No. 2, March 1992.

[6] K. Akita, "Image sequence analysis of real world human motion", Pattern Recognition, Vol. 17, No. 1, 1984, pp. 73-83.

[7] P. Ekman and W. Friesen, Facial Action Coing System, Consulting Psychologist Press, Palo Alto.

[8] R. Forcheimer and O. Fahlander, "Low bitrate coding through animation", PCS 83. 
[9] T. Fukuhara and T. Murakami, "3-D motion estimation of human head for model-based image coding", IEE Proc.-I, Vol. 140, No. 1, February 1993, pp. 26-35.

[10] H. Huang, M. Ouhyoung and J. Wu, "Automatic feature point extraction on a human face in model-based image coding", Opt. Enqrg. Vol. 32, No. 7, July 1993, pp. 157-180.

[11] T. Kurihara and K. Arai, "A transformation method for modeling and animation of human face from photographs", Proc. Computer Animation '91, Springer, Tokyo, 1991, pp. 45-57.

[12] N. Magnenat Thalmann, "Recent techniques in computer animation", Tutorial I Course Notes, Pacific Graphics '93.

[13] N. Magnenat-Thalmann and D. Thalmann, "The direction of synthetic actors in the film Rendez-vous a Montreal", IEEE CG\&A, Vol. 7, No, 12, 1987.

[14] K. Mase and Y. Suenage, "Head reader - A head motion understanding system for better man-machine interaction", IEEE Internat. Conf. on SMC, pp. 970-974.

[15] K. Mase, Y. Watanabe and Y. Suenage, "A real-time head motion detection system", SPIE W orkshop on Sensing and
Reconstruction of Three-Dimensional Objects and Scenes, Vol. 1290, pp. 262-269

[16] M. Nahas, H. Huitric, M. Rioux and J. Domey, "Registered 3D texture image", Proc. Computer Animation '90, Springer, Tokyo, 1990, pp. 81-91.

[17] K. Ohmura, A. Tomono and Y. Kobayashi, "Method of detecting face direction using image processing for human interface", SPIE Visual Comm. Image Process, Vol. 88, pp. 1001-1115.

[18] Y. Suenaga, "Computer vision and graphics for multimedia-based better human interface", Tutorial II Course Notes, Pacific Graphics '93.

[19] D. Terzopoulos and K. Waters, "Physically based facial modeling, analysis, and animation", Visualization Computer Animation, Vol. 1, No. 2, pp. 73-80.

[20] K. Waters, "A muscle model for animating threedimensional facial expression", Proc. SIGGRAPH '87, Vol. 15.

[21] K. Waters and D. Terzopoulos, "Modeling and animation faces using scanned data", J. Visualization Computer Animation, Vol. 2, 1991, pp. 123-128. 